\title{
Scaling in Relativistic Thomas-Fermi Approach for Nuclei
}

\author{
S.K. Patra円, M. Centelles, X. Viñas and M. Del Estal \\ Departament d'Estructura i Constituents de la Matèria, Facultat de Física, \\ Universitat de Barcelona, Diagonal 647, E-08028 Barcelona, Spain
}

\begin{abstract}
By using the scaling method we derive the virial theorem for the relativistic mean field model of nuclei treated in the Thomas-Fermi approach. The Thomas-Fermi solutions statisfy the stability condition against scaling. We apply the formalism to study the excitation energy of the breathing mode in finite nuclei with several relativistic parameter sets of common use.
\end{abstract}

PACS: 21.60.-n; 24.30.Cz; 21.30.Fe; 21.65.+f

Keywords: relativistic mean field; scaling; virial theorem; giant resonances; nuclear incompressibility; Thomas-Fermi theory

E-mail address: mario@ecm.ub.es (M. Centelles)

\footnotetext{
${ }^{1}$ Present address: Institute of Physics, Sachivalaya Marg, Bhubaneswar-751 005, India
} 
The relativistic mean field (RMF) treatment of Quantum Hadrodynamics [1], 2] has proven to be very useful for describing different properties of nuclei along the periodic table. The simplest model, the linear $\sigma-\omega$ model of Walecka [3], explains the nuclear force in terms of the exchange of $\sigma$ and $\omega$ mesons. It is known that the value of the nuclear matter incompressibility is unreasonably high in this linear model $\left(K_{\mathrm{nm}} \sim 550 \mathrm{MeV}\right)$. The problem can be cured by introducing cubic and quartic self-interactions of the $\sigma$ meson [4], and the model can be refined by adding an isovector $\rho$ meson. Current non-linear parameter sets, such as the NL3 set [5], give ground-state energies and densities in excellent agreement with the experimental data, not only for magic nuclei but also for deformed nuclei and for nuclei far from the stability line.

The scaling method has been often employed to derive the virial theorem in the nonrelativistic framework [6], e.g., for nuclear effective interactions such as the Skyrme force [7]. It has also been applied in calculations of nuclear collective excitations like the breathing mode (isoscalar giant monopole resonance) [0]. Relativistic generalizations of the virial theorem obtained by use of the scaling method exist for particles in external potentials [8, 9]. In the RMF model of nuclei the mean field potentials are generated self-consistently. Owing to the meson-exchange nature of the relativistic model one has to deal with finite range forces, which renders the scaling more involved than for zero-range Skyrme forces. Moreover, in contrast to the non-relativistic situation, there exist two different densities, namely the baryon and the scalar density, in accordance with the fact that one has two types of fields, the vector and the scalar field.

In this letter we shall make use of the principle of scale invariance to obtain the virial theorem for the RMF theory by working in the Thomas-Fermi approximation. We shall include non-linear self-couplings of the scalar field and shall deal with spherical finite nuclei. The second derivative of the scaled energy with respect to the scaling parameter, the so-called restoring force, turns out to be positive (stability condition) in the Thomas-Fermi calculations. Thus we are able to apply the method to compute the excitation energy of the isoscalar giant monopole resonance in finite nuclei with realistic parameter sets of the relativistic model. 
The meson field equations of the non-linear $\sigma-\omega$ model are [1, 国]

$$
\begin{aligned}
\left(\Delta-m_{\mathrm{s}}^{2}\right) \phi & =-g_{\mathrm{s}} \rho_{\mathrm{s}}+b \phi^{2}+c \phi^{3} \\
\left(\Delta-m_{\mathrm{v}}^{2}\right) V & =-g_{\mathrm{v}} \rho \\
\left(\Delta-m_{\rho}^{2}\right) R & =-g_{\rho} \rho_{3} \\
\Delta \mathcal{A} & =-e \rho_{\mathrm{p}} .
\end{aligned}
$$

Here $\rho=\rho_{\mathrm{p}}+\rho_{\mathrm{n}}$ is the baryon density, $\rho_{3}=\frac{1}{2}\left(\rho_{\mathrm{p}}-\rho_{\mathrm{n}}\right)$ is the isovector density, and $\rho_{\mathrm{s}}$ is the scalar density. The meson fields $\phi, V$ and $R$ are associated with the $\sigma, \omega$ and $\rho$ mesons, respectively, and $\mathcal{A}$ is the Coulomb field. It is understood that the densities and fields are local quantities that depend on position, even if we do not make it explicit. Units are $\hbar=c=1$.

Taking into account the above field equations, the relativistic energy density of a finite nucleus in Thomas-Fermi approximation can be written as [1, [4]

$$
\mathcal{H}=\mathcal{E}+\frac{1}{2} g_{\mathrm{s}} \phi \rho_{\mathrm{s}}^{\mathrm{eff}}+\frac{1}{3} b \phi^{3}+\frac{1}{4} c \phi^{4}+\frac{1}{2} g_{\mathrm{v}} V \rho+\frac{1}{2} g_{\rho} R \rho_{3}+\frac{1}{2} e \mathcal{A} \rho_{\mathrm{p}}
$$

in terms of the nucleon energy density

$$
\mathcal{E}=\sum_{q} \frac{1}{8 \pi^{2}}\left[k_{\mathrm{F} q} \epsilon_{\mathrm{F} q}^{3}+k_{\mathrm{F} q}^{3} \epsilon_{\mathrm{F} q}-m^{* 4} \ln \frac{k_{\mathrm{F} q}+\epsilon_{\mathrm{F} q}}{m^{*}}\right]
$$

and of $g_{\mathrm{s}} \rho_{\mathrm{s}}^{\mathrm{eff}}=g_{\mathrm{s}} \rho_{\mathrm{s}}-b \phi^{2}-c \phi^{3}$, where

$$
\rho_{\mathrm{s}}=\frac{\partial \mathcal{E}}{\partial m^{*}}=\sum_{q} \frac{m^{*}}{2 \pi^{2}}\left[k_{\mathrm{F} q} \epsilon_{\mathrm{F} q}-m^{* 2} \ln \frac{k_{\mathrm{F} q}+\epsilon_{\mathrm{F} q}}{m^{*}}\right]
$$

is the scalar density and $m^{*}=m-g_{\mathrm{s}} \phi$ is the nucleon effective mass. For each kind of nucleon $(q=\mathrm{n}, \mathrm{p})$ the local Fermi momentum $k_{\mathrm{F} q}$ is defined by $k_{\mathrm{F} q}=\left(3 \pi^{2} \rho_{q}\right)^{1 / 3}$, while $\epsilon_{\mathrm{F} q}=\sqrt{k_{\mathrm{F} q}^{2}+m^{* 2}}$.

The virial theorem relates the kinetic and potential energy components of the energy in certain circumstances. This theorem results from homogeneity properties of the kinetic and potential energy components of $\mathcal{H}$ with respect to a scaling transformation that preserves the normalization. One such normalized scaled version of the baryon density is

$$
\rho_{\lambda}(\boldsymbol{r})=\lambda^{3} \rho(\lambda \boldsymbol{r})
$$


where $\lambda$ is an arbitrary scaling parameter. Accordingly, the local Fermi momentum changes as

$$
k_{\mathrm{F} q \lambda}(\boldsymbol{r})=\left[3 \pi^{2} \rho_{q \lambda}(\boldsymbol{r})\right]^{1 / 3}=\lambda k_{\mathrm{F} q}(\lambda \boldsymbol{r}) .
$$

The meson fields and the Coulomb field are also modified by the scaling due to the selfconsistent equations (11)-(四), which will relate the scaled fields to the scaled densities. Unfortunately, the meson fields do not scale according to simple power laws of $\lambda$ because of the finite-range character of the meson interactions. This is most apparent for the scalar field $\phi$, since the scalar density in the source term of Eq. (11) transforms not only due to the scaling of $k_{\mathrm{F} q}$ but also of $\phi$ itself (or $m^{*}$ ), see Eq. (17) for $\rho_{\mathrm{s}}$. For reasons that will become clear immediately, we shall write the scaled effective mass $m_{\lambda}^{*}(\boldsymbol{r})=m-g_{\mathrm{s}} \phi_{\lambda}(\boldsymbol{r})$ in the form

$$
m_{\lambda}^{*}(\boldsymbol{r}) \equiv \lambda \tilde{m}^{*}(\lambda \boldsymbol{r})
$$

The quantity $\tilde{m}^{*}$ carries an implicit dependence on $\lambda$ apart from the parametric dependence on $\lambda \boldsymbol{r}$.

On account of Eqs. (9) and (10) the scaled form of $\mathcal{E}$ reads $\mathcal{E}_{\lambda}(\boldsymbol{r})=\lambda^{4} \mathcal{E}\left[k_{\mathrm{F} q}(\lambda \boldsymbol{r}), \tilde{m}^{*}(\lambda \boldsymbol{r})\right] \equiv$ $\lambda^{4} \tilde{\mathcal{E}}(\lambda \boldsymbol{r})$, while the scaled scalar density reads $\rho_{\mathrm{s} \lambda}(\boldsymbol{r})=\lambda^{3} \rho_{\mathrm{s}}\left[k_{\mathrm{F} q}(\lambda \boldsymbol{r}), \tilde{m}^{*}(\lambda \boldsymbol{r})\right] \equiv \lambda^{3} \tilde{\rho}_{\mathrm{s}}(\lambda \boldsymbol{r})$. The tilded quantities $\tilde{\mathcal{E}}$ and $\tilde{\rho}_{\mathrm{s}}$ are given by Eqs. (6) and (7) after replacing $m^{*}$ by $\tilde{m}^{*}$. Note the usefulness of (10) to be able to put the transformed densities $\mathcal{E}_{\lambda}$ and $\rho_{\mathrm{s} \lambda}$ into the above compact form. This way, for the scaled total energy density $\mathcal{H}_{\lambda}$ we obtain

$$
\mathcal{H}_{\lambda}=\lambda^{3}\left[\lambda \tilde{\mathcal{E}}+\frac{1}{2} g_{\mathrm{s}} \phi_{\lambda} \tilde{\rho}_{\mathrm{s}}^{\mathrm{eff}}+\frac{1}{3} \frac{b}{\lambda^{3}} \phi_{\lambda}^{3}+\frac{1}{4} \frac{c}{\lambda^{3}} \phi_{\lambda}^{4}+\frac{1}{2} g_{\mathrm{v}} V_{\lambda} \rho+\frac{1}{2} g_{\rho} R_{\lambda} \rho_{3}+\frac{1}{2} e \mathcal{A}_{\lambda} \rho_{\mathrm{p}}\right]
$$

with the definition $g_{\mathrm{s}} \tilde{\rho}_{\mathrm{s}}^{\mathrm{eff}}=g_{\mathrm{s}} \tilde{\rho}_{\mathrm{s}}-b \phi_{\lambda}^{2} / \lambda^{3}-c \phi_{\lambda}^{3} / \lambda^{3}$.

The scaled energy is stationary for $\lambda=1$ (which leads to the virial theorem):

$$
\begin{aligned}
0= & {\left[\frac{\partial}{\partial \lambda} \int \frac{d(\lambda \boldsymbol{r})}{\lambda^{3}} \mathcal{H}_{\lambda}(\boldsymbol{r})\right]_{\lambda=1} } \\
= & \int d \boldsymbol{r}\left[\tilde{\mathcal{E}}-\tilde{m}^{*} \tilde{\rho}_{\mathrm{s}}-\frac{b}{\lambda^{4}} \phi_{\lambda}^{3}-\frac{3}{4} \frac{c}{\lambda^{4}} \phi_{\lambda}^{4}-\frac{1}{2} g_{\mathrm{s}} \tilde{\rho}_{\mathrm{s}}^{\mathrm{eff}} \frac{\partial \phi_{\lambda}}{\partial \lambda}+\frac{1}{2} g_{\mathrm{s}} \phi_{\lambda} \frac{\partial \tilde{\rho}_{\mathrm{s}}^{\mathrm{eff}}}{\partial \lambda}\right. \\
& \left.+\frac{1}{2} g_{\mathrm{v}} \rho \frac{\partial V_{\lambda}}{\partial \lambda}+\frac{1}{2} g_{\rho} \rho_{3} \frac{\partial R_{\lambda}}{\partial \lambda}+\frac{1}{2} e \rho_{\mathrm{p}} \frac{\partial \mathcal{A}_{\lambda}}{\partial \lambda}\right]_{\lambda=1} .
\end{aligned}
$$


Here we have used $\partial \tilde{\mathcal{E}} / \partial \lambda=\tilde{\rho}_{\mathrm{s}} \partial \tilde{m}^{*} / \partial \lambda\left(\right.$ as $\left.\tilde{\rho}_{\mathrm{s}}=\partial \tilde{\mathcal{E}} / \partial \tilde{m}^{*}\right)$ and, from the definition of $\tilde{m}^{*}$,

$$
\frac{\partial m_{\lambda}^{*}}{\partial \lambda}=\tilde{m}^{*}+\lambda \frac{\partial \tilde{m}^{*}}{\partial \lambda}=-g_{\mathrm{s}} \frac{\partial \phi_{\lambda}}{\partial \lambda} .
$$

Let us exemplify the calculation of the derivatives of the scaled fields with respect to $\lambda$ with the omega field $V_{\lambda}$. It fulfils the scaled Klein-Gordon equation $\left(\Delta_{\boldsymbol{u}}-m_{\mathrm{v}}^{2} / \lambda^{2}\right) V_{\lambda}(\boldsymbol{u})=$ $-\lambda g_{\mathrm{v}} \rho(\boldsymbol{u})$, where we have used Eq. (8) for $\rho_{\lambda}$ and have switched to the coordinate $\boldsymbol{u}=\lambda \boldsymbol{r}$. On differenciating this equation with respect to $\lambda$ we have

$$
\left(\Delta_{\boldsymbol{u}}-\frac{m_{\mathrm{v}}^{2}}{\lambda^{2}}\right) \frac{\partial V_{\lambda}}{\partial \lambda}=-g_{\mathrm{v}} \rho-\frac{2 m_{\mathrm{v}}^{2}}{\lambda^{3}} V_{\lambda}
$$

If one now sets $\lambda=1$ the solution of this equation provides $\partial V_{\lambda} /\left.\partial \lambda\right|_{\lambda=1}$. Nevertheless, for our purposes it is more useful to multiply both sides of (14) by $V_{\lambda}$, integrate over the space and then use Green's identity on the left hand side. This way it is straightforward to get

$$
\frac{1}{2} \int d \boldsymbol{u} g_{\mathrm{v}} \rho \frac{\partial V_{\lambda}}{\partial \lambda}=\int d \boldsymbol{u}\left[\frac{1}{2 \lambda} g_{\mathrm{v}} \rho V_{\lambda}+\frac{1}{\lambda^{4}} m_{\mathrm{v}}^{2} V_{\lambda}^{2}\right]
$$

which at $\lambda=1$ is just one of the contributions we need in Eq. (12). Analogous results are found for the rho and Coulomb fields (with a zero mass for the latter). In the case of the scalar field additional terms appear due to the fact that the scalar density itself is a function of the scalar field. Following the same steps as above, from the scaled field equation $\left(\Delta_{\boldsymbol{u}}-m_{\mathrm{s}}^{2} / \lambda^{2}\right) \phi_{\lambda}(\boldsymbol{u})=-\lambda g_{\mathrm{s}} \tilde{\rho}_{\mathrm{s}}^{\text {eff }}(\boldsymbol{u})$ one easily arrives at

$$
\left(\Delta_{\boldsymbol{u}}-\frac{m_{\mathrm{s}}^{2}}{\lambda^{2}}\right) \frac{\partial \phi_{\lambda}}{\partial \lambda}=-g_{\mathrm{s}} \tilde{\rho}_{\mathrm{s}}^{\mathrm{eff}}-\lambda g_{\mathrm{s}} \frac{\partial \tilde{\rho}_{\mathrm{s}}^{\mathrm{eff}}}{\partial \lambda}-\frac{2 m_{\mathrm{s}}^{2}}{\lambda^{3}} \phi_{\lambda}
$$

whence

$$
\int d \boldsymbol{u}\left[-\frac{1}{2} g_{\mathrm{s}} \tilde{\rho}_{\mathrm{s}}^{\mathrm{eff}} \frac{\partial \phi_{\lambda}}{\partial \lambda}+\frac{1}{2} g_{\mathrm{s}} \phi_{\lambda} \frac{\partial \tilde{\rho}_{\mathrm{s}}^{\mathrm{eff}}}{\partial \lambda}\right]=\int d \boldsymbol{u}\left[-\frac{1}{2 \lambda} g_{\mathrm{s}} \tilde{\rho}_{\mathrm{s}}^{\mathrm{eff}} \phi_{\lambda}-\frac{1}{\lambda^{4}} m_{\mathrm{s}}^{2} \phi_{\lambda}^{2}\right] .
$$

From substitution of Eqs. (15) and (17) (and of the corresponding results for the rho and Coulomb fields) into Eq. (12) the virial theorem for the non-linear $\sigma-\omega$ model becomes

$$
\begin{aligned}
0= & \int d \boldsymbol{r}\left[\mathcal{E}-m^{*} \rho_{\mathrm{s}}-\frac{1}{2} g_{\mathrm{s}} \phi \rho_{\mathrm{s}}-m_{\mathrm{s}}^{2} \phi^{2}-\frac{1}{2} b \phi^{3}-\frac{1}{4} c \phi^{4}+\frac{1}{2} g_{\mathrm{v}} V \rho+m_{\mathrm{v}}^{2} V^{2}\right. \\
& \left.+\frac{1}{2} g_{\rho} R \rho_{3}+m_{\rho}^{2} R^{2}+\frac{1}{2} e \mathcal{A} \rho_{\mathrm{p}}\right] .
\end{aligned}
$$


Actually, introducing the kinetic energy density $\tau$ we have $\mathcal{E}-m^{*} \rho_{\mathrm{s}}=\tau+m \rho-m \rho_{\mathrm{s}}$, which makes more obvious the kinetic energy component in the virial theorem. Using Eq. (18) to eliminate $\mathcal{E}$ from the expression of the relativistic energy density $\mathcal{H}$, the RMF energy of a nucleus takes the remarkably simple form

$$
\int d \boldsymbol{r}[\mathcal{H}-m \rho]=\int d \boldsymbol{r}\left[m\left(\rho_{\mathrm{s}}-\rho\right)+m_{\mathrm{s}}^{2} \phi^{2}-m_{\mathrm{v}}^{2} V^{2}-m_{\rho}^{2} R^{2}+\frac{1}{3} b \phi^{3}\right]
$$

where we have subtracted the nucleon rest mass contribution. This expression shows very clearly the relativistic mechanism for nuclear binding. It stems from the cancellation between the scalar and vector potentials and from the difference between the scalar and the baryon density (i.e., from the small components of the wave functions). Equations (18) and (19) are satisfied not only by the Thomas-Fermi solutions, but also by the ground-state densities and meson fields obtained from a quantal Hartree calculation. Of course, the energy stationarity condition against dilation of the RMF problem must be fulfilled by any approximation scheme utilized to solve it.

As a further application of the method we shall use it in calculations of the isoscalar giant monopole resonance (ISGMR). It is customary to write the excitation energy of the ISGMR as

$$
E_{\mathrm{M}}=\sqrt{\frac{C_{\mathrm{M}}}{B_{\mathrm{M}}}},
$$

where $C_{\mathrm{M}}$ and $B_{\mathrm{M}}$ are called, respectively, the restoring force (or incompressibility of the finite nucleus) and the mass parameter of the monopole vibration. To study $E_{\mathrm{M}}$ in the RMF the authors of Refs. [10, 11] resorted to a local Lorentz boost and the scaling method. Following these works one has

$$
C_{\mathrm{M}}=\frac{1}{A}\left[\frac{\partial^{2}}{\partial \lambda^{2}} \int d \boldsymbol{r} \mathcal{H}_{\lambda}(\boldsymbol{r})\right]_{\lambda=1},
$$

where the scaling parameter $\lambda$ now plays the role of the collective coordinate of the monopole vibration, and

$$
B_{\mathrm{M}}=\frac{1}{A} \int d \boldsymbol{r} r^{2} \mathcal{H}(\boldsymbol{r}),
$$


with $A$ being the mass number of the nucleus. The investigations of Refs. [10, 11] were restricted to the linear $\sigma-\omega$ model, either for nuclear matter [10 or for symmetric and uncharged finite nuclei (with the densities solved in Thomas-Fermi approximation) [11]. It is well known that the surface properties of nuclei cannot be described within the linear model, and hence nor can the overall properties of nuclei.

To compute $C_{\mathrm{M}}$ it is easiest to replace the relations (15) and (17) into the expression of $\partial\left[\int d \boldsymbol{r} \mathcal{H}_{\lambda}(\boldsymbol{r})\right] / \partial \lambda$ and derive again with respect to $\lambda$. After some algebra we obtain the restoring force as

$$
\begin{aligned}
C_{\mathrm{M}}= & \frac{1}{A} \int d \boldsymbol{r}\left[-m \frac{\partial \tilde{\rho}_{\mathrm{s}}}{\partial \lambda}+3\left(m_{\mathrm{s}}^{2} \phi^{2}+\frac{1}{3} b \phi^{3}-m_{\mathrm{v}}^{2} V^{2}-m_{\rho}^{2} R^{2}\right)\right. \\
& \left.-\left(2 m_{\mathrm{s}}^{2} \phi+b \phi^{2}\right) \frac{\partial \phi_{\lambda}}{\partial \lambda}+2 m_{\mathrm{v}}^{2} V \frac{\partial V_{\lambda}}{\partial \lambda}+2 m_{\rho}^{2} R \frac{\partial R_{\lambda}}{\partial \lambda}\right]_{\lambda=1},
\end{aligned}
$$

where

$$
\left.\frac{\partial \tilde{\rho}_{\mathrm{s}}}{\partial \lambda}\right|_{\lambda=1}=\left.\frac{\partial \tilde{\rho}_{\mathrm{s}}}{\partial \tilde{m}^{*}} \frac{\partial \tilde{m}^{*}}{\partial \lambda}\right|_{\lambda=1}=-\frac{\partial \rho_{\mathrm{s}}}{\partial m^{*}}\left[m^{*}+g_{\mathrm{s}} \frac{\partial \phi_{\lambda}}{\partial \lambda}\right]_{\lambda=1} .
$$

The derivatives of the scaled meson fields with respect to $\lambda$ are computed by solving Eqs. (14) and (16) at $\lambda=1$. We have found $C_{\mathrm{M}}$ to be positive for all of the (linear and non-linear) parameter sets we have tested in the Thomas-Fermi calculations. A large part of the final value of $C_{\mathrm{M}}$ (usually far more than a half) is due to the contribution of the term $\partial \tilde{\rho}_{\mathrm{s}} /\left.\partial \lambda\right|_{\lambda=1}$.

In Table 1 we display the calculated Thomas-Fermi excitation energies of the ISGMR, together with the empirical estimate $E_{\mathrm{M}} \sim 80 / A^{1 / 3} \mathrm{MeV}$ [12], for ${ }^{40} \mathrm{Ca},{ }^{90} \mathrm{Zr},{ }^{116} \mathrm{Sn},{ }^{144} \mathrm{Sm}$ and ${ }^{208} \mathrm{~Pb}$. Recent experimental data on the centroid energy of the ISGMR are available for these nuclei [13]. We have employed the non-linear parameter sets NL-Z2 $\left(K_{\mathrm{nm}}=172 \mathrm{MeV}\right)$ [14], NL1 $\left(K_{\mathrm{nm}}=212 \mathrm{MeV}\right)$ [15], NL3 $\left(K_{\mathrm{nm}}=272 \mathrm{MeV}\right)$ [5], NL-SH $\left(K_{\mathrm{nm}}=355 \mathrm{MeV}\right)$ [16] and NL2 $\left(K_{\mathrm{nm}}=399 \mathrm{MeV}\right)$ [17]. These parameter sets have been determined by leastsquares fits to ground-state properties of a few spherical nuclei and are of common use in RMF calculations. From the table one can see that the smaller the mass number, the larger is the monopole energy. The energy of the ISGMR increases with increasing $K_{\mathrm{nm}}$ in the various 
parameter sets. For example, the monopole energy in ${ }^{208} \mathrm{~Pb}$ is $12.3 \mathrm{MeV}$ for NL-Z2 while it is 18.1 MeV for NL2. The dependence on $K_{\mathrm{nm}}$ is roughly linear for each nucleus.

In assuming nuclear matter within a certain volume the authors of Ref. [10] estimated the monopole excitation energy of a finite nucleus as

$$
E_{\mathrm{M}}=\sqrt{\frac{K_{\mathrm{nm}}}{\left\langle r^{2}\right\rangle\left(\epsilon_{\mathrm{Fnm}}+g_{\mathrm{v}} V_{\mathrm{nm}}\right)}},
$$

with $\left\langle r^{2}\right\rangle=\frac{3}{5} R^{2}$ and $R=1.2 A^{1 / 3} \mathrm{fm}$. They evaluated (25) for the linear model of Walecka and found $E_{\mathrm{M}}=160 / A^{1 / 3} \mathrm{MeV}$, which has the correct dependence on the mass number but is twice as large as the empirical value. From Eq. (25) one finds $E_{\mathrm{M}}=92,102,115,132$ and $140 / A^{1 / 3} \mathrm{MeV}$ for the non-linear sets NL-Z2, NL1, NL3, NL-SH and NL2, respectively. Comparing with Table 1, the finite size effects reduce the prediction obtained from nuclear matter by a factor ranging from $\sim 1.4$ in ${ }^{40} \mathrm{Ca}$ to $\sim 1.3$ in ${ }^{208} \mathrm{~Pb}$, rather independently of the parameter set.

The ISGMR has been studied in the time-dependent RMF (TDRMF) theory by Vretenar et al. [18]. We include in Table 1 their results for the energy of the main peaks that appear in the monopole strength distributions of ${ }^{90} \mathrm{Zr}$ and ${ }^{208} \mathrm{~Pb}$. Our scaling results compare very well in the case of ${ }^{208} \mathrm{~Pb}$ for all parameter sets, but give somewhat larger excitation energies for ${ }^{90} \mathrm{Zr}$. It should be mentioned that the Fourier spectrum of ${ }^{90} \mathrm{Zr}$ in the TDRMF calculation is considerably fragmented (specially for the sets with higher $K_{\mathrm{nm}}$ ) and then the determination of the centroid energy is more uncertain [18]. Very recently, it has been demonstrated that the relativistic random phase approximation (RRPA) is equivalent to the small amplitude limit of the TDRMF theory in the no-sea approximation, when pairs formed from the empty Dirac sea states and the occupied Fermi sea states are included in the RRPA [19].

Microscopic calculations of ISGMR energies in nuclei are a valuable source of information on the nuclear compression modulus $K_{\mathrm{nm}}$ [20, 21], which is an important ingredient not only for finite nuclei but also for heavy ion collisions, supernovae and neutron stars. A further inspection of Table 1 shows that the empirical law $E_{\mathrm{M}} \sim 80 / A^{1 / 3} \mathrm{MeV}$ lies between the 
predictions of the NL1 and NL3 sets, as expected from the reasonable value of $K_{\mathrm{nm}}$ in these interactions. On the contrary, $K_{\mathrm{nm}}$ is too high in NL-SH and NL2 and we see that these sets overestimate the empirical curve and the experimental data for all nuclei of Table 1. No RMF parameter set seems capable of reproducing the mass-number dependence of the experimental data over the whole analyzed region, particularly in the lighter nuclei. One should note, however, that our calculation provides a prediction for the mean value or centroid of the excitation energy of the resonance. To establish a link between $K_{\mathrm{nm}}$ and the measured energies the most favourable situation is then met in heavy nuclei, where the experimental strength is less fragmented than in medium and light nuclei [13]. If we only take into account the data of ${ }^{144} \mathrm{Sm}$ and ${ }^{208} \mathrm{~Pb}$, our results of Table 1 suggest that $K_{\mathrm{nm}}$ of a RMF interaction should belong to the range 225-255 MeV. (If we disregard the set NL-Z2, as in Refs. [18, 19], the range is 230-260 MeV.) From their TDRMF and RRPA calculations the authors of Refs. [18, 19. conclude that the value of $K_{\mathrm{nm}}$ should be close to 250-270 MeV. Non-relativistic Hartree-Fock plus RPA analyses using Skyrme and Gogny interactions determine $K_{\text {nm }}$ to be $215 \pm 15 \mathrm{MeV}$ 20, 21], thus lower than in the RMF model.

We have derived the virial theorem for the relativistic nuclear mean field model on the basis of the scaling method and the Thomas-Fermi approximation. In this approach we have calculated for realistic parameter sets of the RMF theory the breathing-mode energy of finite nuclei fully self-consistently (i.e., we did not use a leptodermous expansion of the finite nucleus incompressibility as in some previous studies with the scaling method [22]). The present calculations extend earlier work performed with the linear $\sigma-\omega$ model [10, 11].

The excitation energies of the monopole oscillation turn out to be in good agreement with the outcome of dynamical time-dependent RMF calculations. It has been shown very recently that the relativisitc RPA, with the inclusion of Dirac sea states, amounts to the limit of small amplitude oscillations of the TDRMF theory [19. From the present Thomas-Fermi analysis one can thus conclude that, similarly to the non-relativistic case, also in the relativistic framework the excitation energies obtained with the scaling method simulate the results of 
the random phase approximation.

\section{Acknowledgements}

We thank J. Navarro and Nguyen Van Giai for valuable discussions. Support from the DGICYT (Spain) under grant PB98-1247 and from DGR (Catalonia) under grant 2000SGR-00024 is acknowledged. S.K.P. thanks the Spanish grant SB97-OL174874 for financial support. 


\section{References}

[1] B.D. Serot and J.D. Walecka, Adv. Nucl. Phys. 16 (1986) 1.

[2] B.D. Serot and J.D. Walecka, Int. J. of Mod. Phys. E6 (1997) 515.

[3] J.D. Walecka, Ann. Phys. (N.Y.) 83, 491 (1974).

[4] J. Boguta and A.R. Bodmer, Nucl. Phys. A292 (1977) 413.

[5] G.A. Lalazissis, J. Köning and P. Ring, Phys. Rev. C55 (1997) 540.

[6] For scaling relations and the virial theorem see, e.g., R.G. Parr and W. Yang, DensityFunctional Theory of Atoms and Molecules (Oxford University Press, New York, 1989), and references therein.

[7] O. Bohigas, A. Lane and J. Martorell, Phys. Rep. 51 (1979) 267.

[8] M. Brack, Phys. Rev. D27 (1983) 1950.

[9] W. Lucha and F.F. Schöberl, Phys. Rev. Lett. 64 (1990) 2733.

[10] S. Nishizaki, H. Kurasawa and T. Suzuki, Nucl. Phys. A462 (1987) 689.

[11] Chaoyuan Zhu and Xi-Jun Qiu, J. Phys. G17 (1991) L11.

[12] A. van der Woude, Prog. Part. Nucl. Phys. 18 (1987) 217.

[13] D.H. Youngblood, H.L. Clark and Y.-W. Lui, Phys. Rev. Lett. 82 (1999) 691; D.H. Youngblood, Y.-W. Lui and H.L. Clark, Phys. Rev. C63 (2001) 067301.

[14] M. Bender, K. Rutz, P.-G. Reinhard, J.A. Maruhn and W. Greiner, Phys. Rev. C60 (1999) 034304.

[15] P.-G. Reinhard, M. Rufa, J. Maruhn, W. Greiner and J. Friedrich, Z. Phys. A323 (1986) 13.

[16] M.M. Sharma, M.A. Nagarajan and P. Ring, Phys. Lett. B312 (1993) 377.

[17] S.J. Lee, J. Fink, A.B. Balantekin, M.R. Strayer, A.S. Umar, P.-G. Reinhard, J.A. Maruhn and W. Greiner, Phys. Rev. Lett. 57 (1986) 2916.

[18] D. Vretenar, G.A. Lalazissis, R. Behnsch, W. Pöschl and P. Ring, Nucl. Phys. A621 (1997) 853. 
[19] Zhong-yu Ma, Nguyen Van Giai, A. Wandelt, D. Vretenar and P. Ring, Nucl. Phys. A686 (2001) 173; P. Ring, Zhong-yu Ma, Nguyen Van Giai, D. Vretenar, A. Wandelt and Li-gang Cao, Nucl. Phys. (2001), in press.

[20] J.P. Blaizot, J.F. Berger, J. Dechargé and M. Girod, Nucl. Phys. A591 (1995) 435.

[21] M. Farine, J.M. Pearson and F. Tondeur, Nucl. Phys. A615 (1997) 135.

[22] D. Von-Eiff, J.M. Pearson, W. Stocker and M.K. Weigel, Phys. Rev. C50 (1994), 831; M.V. Stoitsov, M.L. Cescato, P. Ring and M.M. Sharma, J. Phys. G20 (1994) L149; T. v. Chossy and W Stocker, Phys. Rev. C56 (1997) 2518. 
Table 1: Excitation energy of the monopole state (in $\mathrm{MeV}$ ) obtained in the scaling approach by using various relativistic parameter sets (in order of increasing value of the compression modulus $K_{\mathrm{nm}}$ ). The energies of the main peaks found in the time-dependent RMF calculations of Ref. [18] are also shown for ${ }^{90} \mathrm{Zr}$ and ${ }^{208} \mathrm{~Pb}$. The experimental centroid energies are from Ref. [13].

\begin{tabular}{rccccccc}
\hline & NL-Z2 & NL1 & NL3 & NL-SH & NL2 & $80 A^{-1 / 3}$ & Exp. \\
\hline${ }^{40} \mathrm{Ca}$ & 20.5 & 21.2 & 23.5 & 26.6 & 29.5 & 23.4 & $19.2 \pm 0.4$ \\
${ }^{90} \mathrm{Zr}$ & 16.4 & 17.2 & 19.2 & 21.9 & 24.0 & 17.9 & $17.9 \pm 0.2$ \\
$\sqrt{18}$ & & 15.7 & $\sim 18$ & & & & \\
${ }^{116} \mathrm{Sn}$ & 15.1 & 15.9 & 17.7 & 20.3 & 22.3 & 16.4 & $16.1 \pm 0.1$ \\
${ }^{144} \mathrm{Sm}$ & 14.1 & 14.9 & 16.6 & 19.0 & 20.8 & 15.3 & $15.4 \pm 0.3$ \\
${ }^{208} \mathrm{~Pb}$ & 12.3 & 12.9 & 14.5 & 16.6 & 18.1 & 13.5 & $14.2 \pm 0.3$ \\
18 & & 12.4 & 14.1 & 16.1 & 17.8 & & \\
\hline
\end{tabular}

